\section{Re: Levamisole-Adulterated Cocaine: A Case Series}

To the Editor: Morris et a ${ }^{1}$ present several striking cases of likely levamisole-adulterated, cocaine-induced vasculopathy. It is appropriate that they highlight the high index of suspicion needed to diagnose this increasingly common cause of acute, acrally predominant retiform purpura and cutaneous necrosis.

As the authors note, detecting levamisole in blood or urine can prove challenging given its short half-life. In addition, only $2 \%$ to $5 \%$ of the parent drug typically is excreted in the urine, further complicating efforts at detection. $^{2} \mathrm{~A}$ recent report ${ }^{3}$ has described the potential utility of testing for levamisole in hair samples from suspected cases using combined ultra-performance liquid chromatography with tandem mass spectrometry, which may further aid in diagnosis of those patients who have delayed seeking medical attention or who last used cocaine more than 48 hours earlier. ${ }^{4}$

Of note, immunologic work-up can be fruitful. In addition to the variable presence of lupus anticoagulant and antinuclear antibodies, the majority of affected patients demonstrate anticytoplasmic neutrophil antibody (ANCA) positivity (perinuclear ANCA greater than cytoplasmic ANCA). ${ }^{5}$ Myeloperoxidase antibodies often predominate, although proteinase 3 antibodies can also be observed. ${ }^{5}$

A less recognized but important marker of levamisoleadulterated, cocaine-induced vasculopathy is the detection of human neutrophil elastase antibodies in serum, which may be useful both for confirmation of the diagnosis as well as differentiation from other pauci-immune systemic vasculitidies. ${ }^{5}$ It is interesting that an antineu- trophil elastase antibody assay has been developed for use in immunohistochemical staining of human tissues for research purposes, although its application for histopathologic evaluation of skin specimens from patients with this vasculopathy has not yet been reported. Future exploration and validation of this alternative technique may significantly contribute to clinicians' ability to accurately and confidently diagnosis this concerning condition.

Jason P. Lott, MD, MSHP Robert Wood Johnson Clinical Scholars Program Department of Dermatology Yale University School of Medicine New Have, CT jason.lott@yale.edu or jason.lott@gmail.com

\section{References}

1. Morris GW Jr, Mason BC, Harris Sprunger R, Hake Harris H, White LA, Patterson DA. Levamisole-adulterated cocaine: a case series. J Am Board Fam Med 2012;25:531-5.

2. Zhu NY, Legatt DF, Turner AR. Agranulocytosis after consumption of cocaine adulterated with levamisole. Ann Intern Med 2009;150:287-9.

3. Lazareth H, Peytavin G, Polivka L, Dupin N. The hairyprint for levamisole-induced vasculitis. BMJ Case Rep 2012 Aug 8;2012. pii: bcr2012006602.

4. Lynch KL, Dominy SS, Graf J, Kral AH. Detection of levamisole exposure in cocaine users by liquid chromatography-tandem mass spectrometry. J Anal Toxicol 2011;35: 176-8.

5. Pearson T, Bremmer M, Cohen J, Driscoll M. Vasculopathy related to cocaine adulterated with levamisole: a review of the literature. Dermatol Online J. 2012;18:1.

doi: 10.3122/jabfm.2013.01.120266

The above letter was referred to the authors of the article in question, who declined to comment. 